\title{
Colonización del
}

\section{Diseño en Argentina} en contraste con la experiencia cubana

\section{Colonization of Design in Argentina in contrast to the Cuban experience}

\author{
Mariana Pittaluga \\ Universidad de Buenos Aires \\ marianapittaluga@gmail.com \\ Buenos Aires, Argentina \\ ORCID 0000-0002-9558-3178
}

Recibido: 18 de junio de 2019 Aprobado: 26 de mayo de 2020

Publicado: 01 de julio de 2020
Resumen

Propongo un recorrido por la historia de la consolidación del Diseño en Argentina a través del concepto de colonización, con relación a la introducción de los modelos y los discursos sobre el Diseño de los países centrales que impactaron en la práctica local. Con la intención de ecualizar el caso argentino con respecto al concepto de colonización, encuentro paradigmático el caso del desarrollo del Diseño en Cuba para plantear la dicotomía entre la introducción de un modelo de Diseño exógeno y la construcción de uno local.

Palabras clave: Colonización, diseño en Argentina, diseño en Cuba, discurso

\section{Abstract}

I propose a journey through the history of the consolidation of Design in Argentina through the concept of colonization, in relation to the introduction of the models and discourses on Design of the central countries that impacted local practice.

With the intention of equalizing the Argentine case with respect to the concept of colonization, I find the case of the development of Design in Cuba paradigmatic to pose the dichotomy between the introduction of an exogenous Design model and the construction of a local one.

Keywords: Colonization, design in Argentina, design in Cuba, discourse 

modelos en Argentina tiene un largo recorrido. Circunstancia asentada por la concepción de la Modernidad. Antes de continuar, se debe señalar que este afuera hace referencia a los países centrales, en contraposición del adentro que alude a Latinoamérica. Hecha esta mención, se debe analizar cómo la Modernidad concentra una visión utópica del progreso en la que se contrapone la idea de la civilización respecto a la idea de la barbarie. Oposición inmortalizada a partir de la obra de Domingo Faustino Sarmiento, figura política y humanista del siglo XIX de Argentina. Ahora bien, ¿cómo llega esta idea de Modernidad a nuestro país? ¿Por qué modernizarse según este parámetro?

Es importante tener presente que la idea de progreso está circunscrita al plano del Iluminismo desarrollado en la segunda mitad del siglo XVIII por la burguesía en Europea. De igual manera que, estas ideas positivistas fueron introducidas en Argentina a partir de la visión particular de diferentes exponentes, entre los cuales se puede destacar a: Mariano Moreno, Esteban Echeverría, Domingo Faustino Sarmiento, Juan B. Alberdi, José Ingenieros, José Ramos Mejía, José Ortega y Gasset, Leopoldo Lugones, entre otros. Esta visión implicó un proceso civilizatorio, y en pos de este se han querido importar modelos sin disolver elementos idiosincráticos. Hecho que puede percibirse en obras como Facundo de Sarmiento o en conferencias sobre El Payador de Lugones en las cuales se visualiza un sincretismo constitutivo de nuestra cultura.

Tal como señala Oporto, la Modernidad consta efectivamente del aspecto del progreso pero trae consigo el del atraso. $Y$ este atraso no tiene que ver con sectores que se hayan rehusado a civilizarse sino con que el proceso de modernización de los sectores dominantes se hizo a costa de la destrucción de las culturas latinoamericanas, africanas y asiáticas (Oporto, 2011, 20).

Hecho que no solo habla de una contraposición entre civilización y barbarie, sino que termina asentando «sociedades desiguales, de desarrollo combinado y siempre vinculadas a los intereses imperiales» (Oporto, $2011,49)$. Retomando esta operación de importación de modelos, cabe visibilizar que un paradigma es un relato, un posicionamiento y una manera de entender al mundo, que en este caso es europea.

Por otra parte, Enrique Dussel (1994) habla del mito de la modernidad. De acuerdo con este paradigma, Europa se ubica en el centro del mundo, preservándose como cultura superior y construyendo al otro como subsumido. Como sostiene García Canclini (2008), la identidad es lo 
articulado por grupos hegemónicos, resultado de un relato que le da coherencia $(2008,17)$. En este mismo sentido también Rorty $(2001,18)$, escribe: «Este proceso de llegar a concebir a los demás seres humanos como 〈uno de nosotros〉 y no como 〈ellos〉 depende de una descripción detallada de cómo son las personas que desconocemos y de una redescripción de cómo somos nosotros».

Sirvan estos planteamientos para analizar el Diseño. Debido a que, al importar modelos de concepción, se importa a través de ellos una ideología. Este acto Bonsiepe (1975) lo refiere como, la práctica del colonialismo cultural. Al subrayar esta práctica es necesario abordar lo que Dussel y Sánchez de Antuñano (1992) observan respecto del trasplante de los logros científicos y tecnológicos, supuestamente universales: el desconocimiento de las necesidades y los requerimientos particulares de los países periféricos, esto con relación a la industria. Refieren cómo en los países centrales la mano de obra es escasa y cara, condición que tratan de suplirla a través de la industria y tecnología. Acorde con esta se pretende como un parámetro universal. Platean procedimientos a los países de la periferia donde la mano de obra es abundante y barata, lo que trae como efecto inmediato el desempleo y una mayor presión social. Pese al hecho de ser un modelo funcional únicamente en países centrales y disfuncional para los países de la periferia, se socavan a estas economías.

Consideraciones sobre el concepto de Diseño
Luego de la segunda guerra mundial, se dio la migración de algunos de los principales exponentes de la Bauhaus como fue la de Ludwig Mies van der Rohe, László Moholy-Nagy, Herbert Bayer, entre otros. Hecho que contribuyó a hacer de los Estados Unidos el epicentro de los siguientes debates sobre diseño. Se puede observar que los referentes en las temáticas son de origen europeo o estadounidense, y a pesar de ello fueron tomados como discursos hegemónicos. Por ejemplo, la modernidad impuesta a través del discurso del Diseño vinculado con la utopía tecnológica progresista. Esto fue posible a partir de la instauración de la producción industrial que perseguían los preceptos desarrollados en la Bauhaus y la Hochschule für Gestaltung ( $\mathrm{HfG}$ ). Además de la preceptuar el discurso histórico en autores como Pevsner, quien impulsó la idea de la Bauhaus como resolución al conflicto arte-industria.

El siglo XX concentró las condiciones históricas en las cuales se asentó la teoría del diseño moderno, la cual se erigió como un discurso dominante. La autora Penny Sparke $(2010,94)$ señala al respecto que: «muchos países en desarrollo sintieron en algún momento la necesidad de adoptarlo, como signo de que ya formaban parte del mundo moderno». La reproducción de estas teorías estuvo a cargo principalmente de exponentes del arte de Latinoamérica. En Argentina se puede aludir a Maldonado quien se formó en Europa y fue representante del movimiento del arte concreto surgido en los años 40. 
Otro caso fue el comienzo de la enseñanza del diseño en Argentina íntimamente relacionado con el modelo de las escuelas alemanas Bauhaus y sobre todo de la $\mathrm{HfG}$. Al respecto, cabe destacar al planteamiento de Verónica Devalle (2009) quien dice que los grandes vectores en la introducción de estas ideas europeas sobre el diseño fueron principalmente Tomás Maldonado y Gui Bonsiepe. En otro escrito, «América Latina, la otra sede de la HfG de Ulm», la autora sostiene a través de un recorrido los vínculos latinoamericanos y los preceptos modernistas de esta escuela.

Una muestra de esta vinculación fue la revista nueva visión creada en 1951 por Tomás Maldonado, Alfredo Hlito y Carlos Méndez Mosquera. Publicación que contó con la colaboración de Max Bill, quien fuera el primer director de la HfG. Este medio impreso se estableció como el principal canal de difusión de las ideas modernistas europeas y como referente de las ideas de la Bauhaus. Se llegó hacer de manifiesto como «el primer discurso habilitante sobre el diseño como problemática general del hábitat pero también como práctica específica» (Devalle, 2016, 58).

En este sentido, Beatriz Sarlo (1988) en Una modernidad periférica: Buenos Aires 1920 y 1930 también propone un recorrido por la introducción de ideas europeas en el campo del arte. También Oscar Terán (2015) realiza un acercamiento a este planteamiento pero de la década de los años 40; da seguimiento de las ideas teóricas durante el primer peronismo y menciona a los representantes de las diferentes corrientes artísticas de este periodo. Otro caso se puede ver desde la arquitectura urbana en Argentina con influencias bauhausianas. La referencia para este caso es el documental Konstruktion Argentina producido por Werner y dirigido por Molnar (2018). En este se informa sobre las relaciones entre arquitectos como Amancio Williams y Walter Gropius. Cabría destacar con relación a este punto, el trabajo de investigación que realiza Joaquín Medina Warmburg sobre la repercusión de las ideas de Gropius en la arquitectura y diseño argentinos.

Todas estas pautas conducen al historiador del diseño Carlos Méndez Mosquera y a su libro Diseño gráfico argentino en el siglo XX. Obra en la que plantea si se puede hablar de la existencia un diseño argentino, a lo cual responde sin titubeos que sí. Sostiene su tesis en la existencia de decenas de diseñadores argentinos. No obstante, en este documento queda claro la afiliación, la relación y la adscripción a un Diseño moderno que la Bauhaus y la HfG entendían sobre este. Se admite explícitamente que las ideas europeas fueron convocadas para: «introducir en la Argentina las nuevas ideas del diseño gráfico internacional no fue tarea fácil, como no la fue introducir el diseño en general» (Méndez, 2015, 36). Con relación a la apertura de la carrera de Diseño Gráfico en la Universidad de Buenos Aires (UBA), Méndez (2015, 20-21) cita a, Guillermo González Ruíz, impulsor de la trayectoria de la gráfica argentina: 
Surgen tres vertientes [...] la primera después de ' 55 tiene su campo operativo en la Facultad de Arquitectura de la Universidad de Buenos Aires, donde Oneto, Méndez Mosquera, Breyer, Le Pera, Oliver, Moller, Janello, Rotzait y otros serán los encargados de demostrar a través de la formación de nuevos arquitectos [que las semillas del Bauhaus habían germinado en Buenos Aires y se iban a expandir posteriormente en Rosario, Mendoza y La Plata].

Es de notar aquello que Penny Sparke (2010) sostiene respecto de la repercusión de la ideología del Movimiento Moderno sobre la teoría y práctica del diseño del siglo XX. Pues refiere en esta un discurso hegemónico, introducido y replicado desde el sistema institucional. Asimismo, la tesis de la visión en Latinoamérica de un Diseño importada y trasplantada. Puede también ser respaldada en las reflexiones a las que llegan Enrique Dussel y Sánchez de Antuñano $(1992,5)$ cuando sostienen al respecto:

En el campo del Diseño Industrial, de reciente creación en el panorama mundial como disciplina, los profesionales continúan formándose en los sistemas pedagógicos de los países del «centro», que han adoptado nuestros centros educativos. Siguiendo estos modelos resulta difícil que de ellos se obtengan resultados que promuevan una tecnología propia, así como el impulso de la pequeña y mediana industria nacional. En la mayoría de los casos y por una falta de ubicación dentro de la realidad concreta, tanto social como productiva, su acción se enfoca a la creación de productos suntuarios y accesorios.

¿Es posible hablar de un Diseño argentino?
Considérese ahora la pregunta ¿es posible hablar de un Diseño argentino? Como podrá inferirse en esta interrogante se plantean diversos presupuestos. Pero esta nace del recorrido hasta este momento expresado, se recordará cómo la práctica del diseño fue incorporada en los países latinoamericanos a partir de una concepción extranjera. Hecha esta salvedad, ¿es posible llamarlo diseño argentino solo por el hecho de desarrollarlo en este país?

Como se afirmó arriba la construcción de las teorías del diseño, sus definiciones y postulados surgieron en un contexto europeo, esencialmente alemán. Luego, fueron adoptadas principalmente por Tomás Maldonado y Gui Bonsiepe, ambos integrantes de la HfG. En concordancia con esta práctica reproducida no habría per se un diseño argentino. Esto se contrapone a la postura de Méndez Mosquera quien afirma su existencia por el hecho de que hay diseñadores que realizan esta práctica. Pero ¿es suficiente este argumento para sustentar tal premisa?

Conforme a la visión de Víctor Margolin, historiador estadounidense del Diseño, expresa que durante la primera mitad del siglo XX el diseño fue incorporado con las perspectivas europeas y estadounidenses en los 
países latinoamericanos; menciona a Cuba, México, Brasil, Chile, Argentina, Colombia, Venezuela y Uruguay; omite a Bolivia, Perú, Paraguay, Ecuador, Nicaragua, Guatemala y Puerto Rico. Es de notar que los alcances de la obra de Margolin, Historia mundial del Diseño, tiene omisiones que resultan paradójicas al título donde figura la palabra mundial. ${ }^{1}$ No obstante, es posible percibir una clara relación entre la industria y el diseño. Además de poder distinguir las influencias estilísticas europeas en los diseñadores y sus diseños, según sus escuelas, sus referentes o sus estudios realizados en Europa o los Estados Unidos. A través de esta obra, se puede llegar a comprender que el presupuesto diseño es puesto en relación y en comparación con un modelo industrial capitalista:

Because so many durable goods came from abroad, even if some were assembled locally, there was little opportunity to develop a profession of industrial design compared to Europe or the United States, where manufacturers with advanced production capabilities relied on designers to create new products for the market.

[Porque muchos bienes provenían de afuera, aun cuando algunos fueran ensamblados localmente, allí había pocas oportunidades para desarrollar la profesión del diseño industrial comparado con Europa o Estados Unidos, donde la manufactura recaía en los diseñadores para crear nuevos productos para el mercado] (Margolin, 2017, 462, traducción propia).

Por otro lado, Gui Bonsiepe y Silvia Fernández compilan en el libro Historia del diseño en América Latina y el Caribe (2008) un panorama de la conformación del diseño de ciertos países. En esta obra se mencionan los casos de: Argentina, Brasil, Colombia, Cuba, Chile, Ecuador, México, Uruguay y Venezuela; se omiten: Bolivia, Perú, Paraguay, Nicaragua, Guatemala y Puerto Rico. Respecto al Diseño argentino, señalan que este fue propiciado por una temprana industrialización y de una fuerte influencia de las experiencias de la Bauhaus y la $\mathrm{HfG}$, además de las intervenciones de Tomás Maldonado y Gui Bonsiepe (confróntese con Devalle, 2009).

Mientras la industria funcione, el Diseño funcionará. Sin embargo, durante la década de los años 90 , fue un periodo de crisis industrial y por ende de crisis en el diseño, específicamente del diseño industrial. Esto debido a que la privatización mermaba la demanda de diseño nacional. Pero esta situación fue distinta en el diseño gráfico. Ya que, las imágenes corporativas cambiaron pues las empresas que eran del estado se privatizaron. Se puede notar cómo los periodos de crisis industrial significaron crisis en el diseño.

1 Es interesante destacar el hecho de las omisiones que nos recuerdan al mismo nombre de América Latina, lo que termina siendo una adopción arbitraria. Puesto que, no entran dentro de esta noción países con raíces, entendiendo a esta palabra por colonizadores, latinas como Canadá y sí lo conforman países angloparlantes como Jamaica, pero con la salvedad de que estos últimos son pobres (Oporto, 2011, 31). 
Si comparamos con el caso uruguayo y chileno en un plano académico, Uruguay tuvo un inicio del Diseño relacionado con el Arte de la mano de Torres García, quien introdujo las ideas de la Modernidad europea. Pero también aparece el nombre de Maldonado y una metodología adscripta al movimiento moderno de la Bauhaus y HfG. En el caso de Chile, este tuvo un proceso similar al de Argentina, sus escuelas fueron dominadas por las experiencias planteadas en Europa, con la participación e influencia de Gui Bonsiepe, quien se instaló en ese país durante varios años.

Por contraste, se puede advertir cómo en aquellos países con costumbres, tradiciones y herencias prehispánicas, los recorridos fueron diferentes. De alguna manera sincrética logró sobrevivir. Sin embargo, los cambios globales en la economía impactaron drásticamente en todos los aspectos culturales, incluido el diseño. Tales fueron los casos en Ecuador, Colombia, Venezuela y México. En particular, del diseño mexicano Enrique Dussel y Jorge Sánchez de Antuñano (1992) realizan un exhaustivo estudio. Plantean el cuestionamiento de la importación de modelos de los países centrales, en un contexto pedagógico como en el diseño. Concluyen que esto deviene en una postura dependiente.

(2) El caso paradigmático: la experiencia cubana
Con la intención de contrastar el caso de Diseño en Argentina con relación al concepto de colonización se encuentra el paradigmático caso de Diseño desarrollado en Cuba. En esta comparación se plantea la dicotomía entre la introducción de un modelo de Diseño exógeno y la construcción de un modelo propio. Es posible distinguir dos periodos en el desarrollo del diseño cubano de mediados del siglo XX. El primer periodo abarca de la década de los años 60 hasta la década de los años 80 realizado con apoyo de la Unión Soviética. El segundo periodo comenzó en la década de los años 90 sin la Unión y con el bloqueo económico y comercial.

Durante el primer periodo fueron establecidos planes, instituciones y escuelas de diseño cercanos a los preceptos de la Modernidad. Esto es, una proximidad alineada con los principios de la Bauhaus y HfG como en el resto de Latinoamérica. Respecto al orden institucional fueron establecidos: el Ministerio de Industria, que fue presidido por Ernesto Guevara; el Ministerio de la Industria Ligera; el Departamento de Muebles de la Dirección de Arquitectura del Ministerio de la Construcción; la Oficina de Estudios y Desarrollo de Productos adscrita al Ministerio de Industria; el Instituto Cubano de Investigación y Orientación de la Demanda Interna con un departamento de diseño y una dirección de equipamiento para la vivienda; la Industria Nacional Productora de Utensilios Domésticos; la Escuela de Diseño Informacional e Industrial (EDII), primera escuela superior de diseño, dependiente originariamente del Ministerio de la Industria Ligera y luego del Ministerio de Cultura y dirigida por el arquitecto Iván Espín; la Oficina Nacional de Diseño Industrial y en el Instituto Superior de Diseño Industrial (ISDI). 
Se estima que esta primera etapa concebida sobre Diseño fue introducida más que desarrollada. Si bien inicialmente fue establecida con los preceptos de la Bauhaus y de la $\mathrm{HfG}$, también se puede distinguir una perspectiva socialista en la que se proyectaba una producción industrial lejana de los intereses neocapitalistas. Esto bien puede observarse cuando Tomás Maldonado lo expreso inicialmente a través del artículo Nosotros y el mundo de las mercancías escrito en 1965, y después vuelve hacer manifiesta su posición respecto a los objetivos del modelo neocapitalista industrial y como este se contraponía a la idea que tenían los proyectistas de Ulm de la siguiente manera:

De pronto, descubríamos los vicios ocultos de los productos «bien diseñados», y también de la filosofía que les había servido de fundamento. De pronto, contábamos, no sin embarazo, que nuestra actividad como proyectistas contribuía a la devoción irracional por las mercancías, cuando nuestro designio originario había sido muy otro: conferir estructura y contenido al entorno humano (Maldonado, 1977, 187-188).

La actividad proyectada no dejaba de ser un modelo generado para y por una sociedad europea en reconstrucción. Desarrollada por un grupo de países centrales. Una de las implicaciones, era la resolución desde de la esfera central para problemáticas de la esfera periférica. Otra es que, la concepción de diseño planteada por estas corrientes mantiene una relación directa y necesaria con la industria.

Como se afirmó arriba, la primera experiencia académica en Cuba se apoyaba en los conceptos de la teoría y la práctica del diseño gestada en Europa. Esto puede saberse además por Bonsiepe (1975) quien es citado por Fernández Uriarte para señalar su participación como invitado a dar un seminario sobre diseño de mobiliario, organizado por el Instituto Cubano de Investigaciones y Orientación de la Demanda Interna en 1972, así como de la colaboración de Tomás Maldonado en 1979 en la EDII:

Los fundadores del ISDI, provenientes en su mayoría de la Facultad de Arquitectura y en particular del Departamento de Diseño Básico, aportaron un hito de continuidad y apropiación de las tesis racionalistas de la Bauhaus y de la Escuela de Ulm (HfG), lo que se manifestó en la enseñanza del Ciclo Básico en las cualidades formales de los productos y en la conceptualización y metodología a seguir. De particular relevancia en este sentido resultó el libro Teoría y práctica del diseño industrial, de Gui Bonsiepe específicamente impreso por el Ministerio de Enseñanza Superior para el Instituto (Fernández Uriarte, 2016, 20).

Se puede deducir esto además por la concepción sobre el Diseño que tenía el que fue director de la EDII, «podemos definir al diseño como el trabajo ( $y$ el cuerpo de teorías, métodos y técnicas), que tiene como fin la preparación anticipada de las especificaciones detalladas para la producción industrial de objetos de consumo» (Espín citado por Fernández Uriarte, 2016, 89). 
Por su parte, Gonzalo Córdoba, diseñador argentino radicado en Cuba desde 1949, fue de gran importancia e influencia en el desarrollo del Diseño Industrial cubano. Es de considerar su opinión cuando se refirió al diseño de su «butaca Guamá» desarrollada en 1961: «debería cumplir la expectativa de llevar la cultura del diseño a la población» (Fernández Uriarte, 2016, 11). En esta subyace la idea de un diseño evangelizador. Un modo de hacer, una metodología y un saber circunscrito de un grupo selecto que se impone, avalado por el carácter revelador del Diseño.

Retomando el punto que hace alusión al segundo periodo, es el de un contexto de crisis en Cuba. A saber, esto repercutió tanto a la esfera industrial y como a la esfera del diseño. El marco teórico del Diseño cubano, era hasta ese momento dependiente de las herramientas de los países centrales. Pero el diseño es redefinido, tanto su práctica como sus preceptos dan un giro. Tras la interrupción de los subsidios de la Unión Soviética, y el cese de las importaciones de Europa del Este, Cuba impulsó un programa denominado ANIR (Asociación Nacional de Innovadores y Racionalizadores). Este consistía en la alfabetización tecnológica y la enseñanza a todos los ciudadanos en la reparación y la modificación de artefactos. A raíz de este programa, fue realizado el manual, Con nuestros propios esfuerzos $(1992,9)$, obra en la que se publican una serie de soluciones de distinta índole:

La recopilación de la información expuesta en este libro se organizó a través de los Estados Mayores Provinciales y Municipales en coordinación con los Comités Municipales del Partido y el Gobierno.

Lamentablemente no todos los municipios enviaron sus experiencias y no todas las fichas que recibió la Editora describían correctamente el objeto de la experiencia [...] Muchas iniciativas quedaron en nuestras manos sin publicar por problemas de espacio y la mayoría por deficiencias en la descripción de las experiencias, imprecisiones e incomprensiones en la lectura.

Es en este contexto donde emergen los discursos críticos. Entre los cuales se encuentra el del grupo Ordo Amoris, el cual organizó una serie de exposiciones de objetos cotidianos creados popularmente. Ernesto Oroza, diseñador cubano y quien formó parte de ese colectivo crítico, desarrolló el concepto de desobediencia tecnológica. Este hace referencia a la producción de objetos populares. Otro de sus conceptos es el de arquitectura de la necesidad, idea que alude a aquellas soluciones arquitectónicas subversivas, pensadas para garantizar la vida en sociedad en una economía deprimida. Utilizó el término para definir un conjunto de acciones creativas cotidianas ocurridas en Cuba durante la década de los años 90. Expresó sobre el concepto desobediencia tecnológica:

Me refiero, entre muchas otras, a la acumulación, la reparación y el reuso. Son prácticas que no parecen muy desobedientes pero que ponen en cuestión radicalmente las lógicas tecnológicas y de consumo de los objetos industriales. 
Entre las lógicas cuestionadas están el ciclo de vida de los objetos, muchas veces preconcebido por el fabricante; $y$ el objeto industrial cerrado, concluido, casi hermético, cuyo diseño no incluye la posibilidad de reparar o de ser intervenido por el usuario.

Es remarcable que el cuestionamiento de los objetos y lógicas industriales se produjo desde una perspectiva artesanal. Eran lógicas y productos industriales revisados desde procesos artesanales y operaciones manuales. Los cubanos diseccionaron la cultura industrial, abrieron todos los cuerpos posibles, repararon y alteraron todo tipo de objetos sin dejarse intimidar por la complejidad técnica ni la escala, como es el caso de los automóviles.

Entonces, llamo Desobediencia Tecnológica a un sistemático irrespeto del individuo en Cuba ante la complejidad y lógicas cerradas y excluyentes de los objetos industriales capitalistas y comunistas (Oroza, 2015, párr., 12-14).

Ante una situación de crisis o a partir de ella fue que el pueblo cubano, desarrolló un sistema propio, endógeno. En este sentido Oroza (2002, 11) sostiene enfáticamente que «On parle, précisément, d'une production de crise qui n'est pas issue d'un choix mais des contraintes ou des résistances de la survie» [Hablamos, precisamente, de una producción de crisis, que no es resultado de una elección sino de condicionantes o por la resistencia de sobrevivir] (traducción propia).

No obstante, ninguna de las características de los objetos de desarrollo cubano se ajustaría a los parámetros modernos del Diseño. Tampoco el hecho de que no existiría un diseño o un diseñador como tal. Por ello es que, Oroza asocia este abordaje cubano a la invención y los bautiza como objetos de la necesidad u objetos reinventados «Je me référe à une maniére différente d'entrer en relation avec les matériaux, le fait créatif et l'appréciation esthétique» [Me refiero a una relación diferente entre los materiales, el hecho creativo y la apreciación estética, traducción propia] (Oroza, 2002, 10, traducción propia). Esto conlleva a pensar que, si el sistema moderno no funciona sin las herramientas de los países centrales, no es un sistema que se pueda trasladar a contextos con otras particularidades, complejidades y recursos. Sin embargo, esto no se ha comprendido en gran parte de Latinoamérica, siempre sostuvo la pata industrial.

Con base en lo expuesto se realiza el siguiente razonamiento: si la definición del Diseño está proyectada a través de un modelo de producción moderno de los países centrales ¿se puede llamar diseño a las producciones desarrolladas fuera de este contexto, como es el caso de los objetos de producción popular que menciona Oroza? Desde una perspectiva moderna, la respuesta sería no. Puesto que, estos objetos populares no son producto de la industria sino de las modificaciones y las resignificaciones, ni son realizados ni son dirigidos por diseñadores. Tampoco se rigen por sus parámetros estéticos de lo moderno y los preceptos de la 
«gute form», (Max Bill citado por Maldonado,1977, 68). Son objetos totalmente diferentes a aquellos de la primera etapa del diseño emergente cubano, diferentes de la vajilla Tezoro que ganó la medalla Gutes Design y que fuera expuesta en Berlín. Esta vajilla es una referencia que respondía a los parámetros hegemónicos del Diseño.

Esta coyuntura en Cuba hizo que esta metodología no pudiera ser sostenida. Sin embargo, de las problemáticas seguían surgiendo diseños como las antenas de televisión resueltas con bandejas metálicas de comida. Esto se considera innovador desde el punto de vista del proceso de diseño. Puesto que, se trata de una respuesta colectiva a una problemática colectiva. Incluso es destacable en términos identitarios, ya que es reconocido como propio por la población cubana.

Se puede clasificar el proceso de diseño cubano de estos artefactos en tres aspectos: reparación, refuncionalización, y reinvención. En esta tríada consta la desobediencia de la cual habla Oroza. Esta metodología se transformó en una posición ideológico-política manifiesta en estos objetos. Se trata de una voz genuinamente cubana. En contrasentido, es la relación y la producción del diseño argentino que reproduce las reglas exógenas de un diseño central. Asimismo, se destaca que los aspectos del proceso de diseño cubano de la década de los años 90, son la muestra de una perspectiva del diseño consciente y responsable.

Cabe preguntar, ¿estos objetos son diseño o no? De acuerdo con la taxonomía, la definición sería, no. Desde la otra perspectiva pueden ser considerados como diseño, no ponderando la forma sino el proceso de pensamiento mediante el cual se ha dado una respuesta a una problemática.

Conclusiones Como se hizo observar, el proceso de modernización en Argentina fue largo y complejo. Se inició simultáneamente con la conformación del Estado y no se puede asegurar que haya finalizado. No obstante, mi advertencia fue sobre la adopción de modelos e ideologías de los países centrales.

A mediados del siglo $X X$, se establecieron gran parte de los preceptos del Diseño como disciplina. En Argentina, se consolidaron estos fundamentos desde una perspectiva europea. Desde este recorrido es que se planteó si habría un Diseño argentino. Con base en el recorrido propuesto, se puede decir que se encontró una práctica realizada a partir de modelos hegemónicos exógenos. Esto en contraposición con el caso cubano, debido a que ahí han repensado un sistema de producción. Esto refuerza lo planteado con respecto al caso argentino.

Para concluir, realizo a manera de reflexión los siguientes cuestionamientos: en primer lugar, y con relación al caso de la vajilla Tezoro lleva 
a preguntarse cómo es que la producción de su diseño realizado en un país periférico puede asimilarse tanto a la producción del diseño planteado desde los preceptos de los países centrales; en segundo lugar, al hablar de desobediencia y de innovación en la producción objetual cubana ¿no responde a los conceptos del diseño responsable?; en tercer lugar, ¿son las nuevas tendencias de diseño conscientes y responsables una forma fagocitada por la cultura de diseño hegemónico ajustada a un mercado?; en cuarto lugar, ¿dónde reside la diferencia entre los objetos de producción colectiva cubanos y los de experiencias como Sea Chair? Por último, ¿es posible pensar en una perspectiva de diseño propia que abarque y dé cuenta de las particularidades tanto sociales como culturales de su entorno?

Con esta última interrogante me gustaría señalar cómo este tipo de experiencias se comprenden como algo exótico y superficial de la cultura local. En este sentido, puedo mencionar el proyecto Guajira, el cual es una exposición de mobiliario diseñado por Carilyn de la Vega, diseñadora industrial y profesora Instituto Superior de Diseño que en colaboración con el diseñador gráfico e ilustrador Carlos Pérez Zamora llevaron a cabo en Cuba en el mes de abril de 2018. En esta muestra se expuso el diseño de piezas basado en los sombreros tejidos tradicionales de los campesinos cubanos. Si bien la confección y la inspiración están basadas en las costumbres y las maneras de realización locales, desde mi opinión, es un trabajo que se queda como una anécdota y que destaca por un aspecto meramente formal. Esta exposición vista desde la hegemonía es ponderada por su exotismo. Cabe destacar que, este proyecto fue desarrollado con el apoyo de la Embajada de Noruega. Es un diseño tomado a modo de fórmula: un material, una técnica y una anécdota. No se trata de una experiencia que resuelva de forma integral una problemática, que contribuya en algún aspecto social o en la visibilización de alguna complejidad cultural a diferencia de los artefactos expuestos por Oroza. Es decir que, se trata de un caso que resuelve una cuestión morfológica que podemos ubicar en la esfera del buen diseño dominante. Esta experiencia no es distinta a la de la butaca Guamá. En realidad, se podría ver el mobiliario Guajira como una introducción de un aspecto local a una concepción hegemónica del diseño. ${ }^{2}$

2 Se puede encontrar experiencias similares en este aspecto, como la del diseño de un taburete desarrollado por el estudio británico H Furniture, con telares típicos mexicanos. La colección fue llamada «Loam Collection», y la serie consiste en una silla «Loom Chair» y un taburete «Loom Stool», véase en: https://www.hfurniture.co/product_collection/loom-collection/ 
Referencias Bonsiepe, G. (1975). Diseño Industrial. Artefacto y Proyecto. Madrid: Alberto Corazón Editor.

Bonsiepe, G. y Fernández, S. (2008). Historia del diseño en América Latina y el Caribe. San Pablo: Editora Blucher.

Canclini García, N. (2008). Culturas híbridas. Estrategias para entrar y salir de la modernidad. Buenos Aires: Paidós.

Devalle, V. (2009). La travesía de la forma. Buenos Aires: Paidós.

Devalle, V. (2016). América Latina, la otra sede de la HfG de Ulm. RChD: creación y pensamiento. 1(1), 53-63.

Dussel, E. (1994). 1492: el encubrimiento del otro: hacia el origen del mito de la modernidad. La Paz: Plural editores.

Dussel, E. y Sánchez de Antuñano, J. (1992). Cuestionamiento de la Situación actual del diseño y la tecnología. Contra un Diseño Dependiente: un modelo para la autodeterminación nacional. México: Universidad Autónoma Metropolitana.

Con nuestros propios esfuerzos. (1992). La Habana: Editora Verde Olivo.

Fernández Uriarte, L. (Coord.) (2016). Modernidad, identidad y valor social: el diseño en Cuba de 1960-2000. La Habana: Ediciones Forma.

Maldonado, T. (1977). Vanguardia y racionalidad. Barcelona: Gustavo Gili.

Margolin, V. (2017). World History of Design. Prehistoric Times to World War I. New York: Bloomsbury.

Medina Warmburg, J. (2017). El mundo como artefacto Tomás Maldonado en el foco del diseño ambiental (1966-1972). Ra. Revista de Arquitectura, 25-38.

Medina Warmburg, J. (2018). Walter Gropius, Proclamas de Modernidad. Escritos y conferencias, 1908-1934. Barcelona: Reverte.

Méndez Mosquera, C. (2015). Diseño gráfico argentino en el siglo XX. Buenos Aires: Ediciones Infinito.

Oporto, M. (2011). De Moreno a Perón. Buenos Aires: Planeta.

Oroza, E. (14 de febrero de 2015). Desobediencia Tecnológica. Preguntas por Jenny Marder. [Technological Disobedience, blog]. Recuperada de http:// www.technologicaldisobedience.com/2015/02/14/desobedienciatecnologica-preguntas-por-jenny-marder/

Oroza, E. y Bozzi de, P. (2002). Objets réinventés. París: Éditions Alternatives.

Pevsner, N. (1963). Pioneros del diseño moderno. De William Morris a Walter Gropius. Buenos Aires: Ediciones Infinito.

Rorty, R. (2001). Contingencia, ironía y solidaridad. Barcelona: Paidós.

Sarlo, B. (1988). Una modernidad periférica: Buenos Aires 1920 y 1930. Buenos Aires: Nueva Visión.

Sparke, P. (2010). Diseño y cultura, una introducción. Desde 1900 hasta la actualidad. Barcelona: Gustavo Gili.

Terán, O. (2015). Historia de las ideas en la Argentina. Diez lecciones iniciales, 1810-1980. Buenos Aires: siglo veintiuno.

Werner, D. A. (Productor), Molnar, F. (Director). (2018). Konstruktion Argentina. [Documental]. Argentina: INCAA / Werner Cine. 


\section{Sobre la autora Mariana Pittaluga}

Doctoranda en Arte Contemporáneo Latinoamericano por la Universidad Nacional de La Plata. Especialista en Teoría del Diseño por la Facultad de Arquitectura, Diseño y Urbanismo, Universidad de Buenos Aires (FADU-UBA). Diseñadora Gráfica por la FADU-UBA. Diplomatura de Posgrado en Cultura y Sociedad del Instituto de Altos Estudios Sociales por la Universidad Nacional de San Martín. Posgrado en Identidad Corporativa por la FADU-UBA. Diplomatura en Pensamiento Complejo en la Multiversidad del Mundo Real en México.

Profesora titular de la Maestría de Tipografía de la FADU-UBA. Fue integrante del equipo de investigación UBACyT Proyecto A 406 con Sede en el Instituto de Arte Americano de la FADU-UBA. Miembro del Comité de Dictaminación de la revista Zincografía, perteneciente al departamento de Proyectos de Comunicación del Centro Universitario de Arte, Arquitectura y Diseño de la universidad de Guadalajara. Se especializa en Branding y Diseño Editorial. Trabaja como profesional independiente, y se desempeña como Coordinadora de la Oficina de Diseño de la Editorial Jusbaires del Poder Judicial de la Ciudad de Buenos Aires. 